\title{
Implementation of an On-Line Archive for Retrieval of Laboratory Results for Outpatients
}

\author{
Daniel M. Bloch, M.D., Bruce Barnes, B.S., M.T. \\ (A.S.C.P.), and Steven Pade, B.S.
}

\begin{abstract}
An on-line archive of laboratory results designed specifically for ambulatory care areas is described. It is a direct result of paper-flow problems in clinics, which were manifested by numerous phone calls to the laboratory for test results performed up to 10 months ago, and requiring as much as 30 minutes each for retrieval. The archive is incorporated into the result inquiry program of our laboratory computer and is transparent to the user. Utilization of this system by our personnel saves up to 12 hours of work each day. By capturing in a separate file the patient location of each call for outpatient results we are able to identify the clinics that use the telephone for retrieval of test results.
\end{abstract}

\section{INTRODUCTION}

Computers have been used in the clinical laboratory for over 15 years. Their main purpose is to enhance communication of test results from the laboratory to the clinician. The objective of laboratory computing for hospitalized patients is the delivery of test results in a timely manner. ${ }^{1}$ For ambulatory care patients, on the other hand, speed is not usually as important as the assurance that test results are available when the patient returns to clinic, which in many instances occurs weeks to months later. Laboratory computers are designed to treat both areas in a parallel fashion because very little difference exists within the laboratory in how specimens and results are handled. Our experience over the last 5 years indicates that it is much more difficult to achieve the same degree of success in the ambulatory care setting than for the hospital because they are totally different functional entities.

As a result of paper-flow problems in the ambulatory care facility, it is not uncommon for the test results to be unavailable to clinicians even though the reports were printed and distributed. The outcome is that the Laboratory Data Center at The University

From the Department of Pathology, The University of Michigan Medical Center, Ann Arbor, Michigan 48109.

$\uparrow$ This paper was presented at the SAMS/SCM Conference on Computers in Ambulatory Medicine, November 1981, and is republished here with the permission of AAMSI. 
of Michigan receives many phone calls from the clinics. For patients who have returned to clinic within several weeks, answering these phone inquiries was never a major problem. However, if the time was longer, results were no longer in the system due to limitations in file size, and another means of retrieval was required. Several schemes were tried, including retrieval from hard copy and microfische, however, these were found to be too cumbersome and time-consuming. Not until we designed and implemented an online archive for laboratory results were we able to deal with this special need of the ambulatory care area.

This paper describes an on-line archive for laboratory results designed specifically to address the unique problems of information flow in the ambulatory care area.

\section{MATERIALS AND METHOD}

The University of Michigan Department of Pathology has a turnkey laboratory computer system, the details of which have been described elsewhere. ${ }^{1,2}$ The laboratories perform over 5.5 million tests yearly. There are nearly 320,000 outpatient visits per year at 50 discrete locations, which are spread out in four buildings over a 1-mile area. Specimens are obtained at or delivered to a centralized outpatient blood-drawing area, where they are accessioned into the laboratory computer and passed to the laboratory for analysis and input of results. Interim and cumulative reports are printed frequently and are delivered to the clinics and nursing stations by various means. After a specified time period without additional laboratory activity, a final report is printed for medical records and the data are purged to magnetic tape.

The problems of information flow in the ambulatory care area that compelled us to design an on-line archive are considered in the results and discussion section that follows.

To solve the problems described above, we designed the on-line archive for the laboratory computer that we had since 1976. This archive occupies a dedicated 55megabyte formatted SMD disk drive costing $\$ 17,000$. The software for the archive is written in FORTRAN IV and required 2 months to design, code, and test. Access to the archive is integrated into the standard result inquiry program of the system and is therefore transparent to the user. Results from up to 10 months ago are available in 2 to 3 seconds by either specifying a specific number of days back to search or by using the start-end option to define a time window. The computer searches the active patient files and then automatically accesses the archive if necessary. After a specified number of days without additional laboratory activity, when the final report is to be printed and the data are to be purged onto magnetic tape, the active files are automatically converted to the archive file format. During this process all unnecessary file structure and data are eliminated, allowing compression of the file. The minimum data required for the archive are patient name, registration number, birth date, sex, location, test name, specimen collection date/ time, and test results, including high/low flags. Other items such as test request and verification time/date, as well as technologist identification, being available in other offline archives and of no value to the clinician, are eliminated. The program automatically determines the amount of new disk space required and allocates it by elimination of only the oldest data in the archive. Thus, the archive is completely self-maintaining and requires no computer operator intervention for file maintenance. Every night after this 
conversion and reallocation of space the appropriate files are written onto the archive disk. Approximately 10 months of data are stored on-line, which is equivalent to 5 million test results. Whenever the result inquiry program is called, the time and the patient location can be captured and stored in a separate file. The utility of this type of information is discussed below.

\section{RESULTS AND DISCUSSION}

Implementation of this on-line archive system for laboratory results immediately eliminated the need for tedious manual searches of our paper or microfische archives in order to answer phone inquiries from the outpatient clinics for results up to 10 months ago. Since it was integrated into the result inquiry program, no additional steps are required of the laboratory assistants who answer phone inquiries. Except for the option of specifying a time window, it is competely transparent to the user. Physicians in the ambulatory care areas now receive instant response, and patients are not required to wait for previous test results before being seen. These previously required manual searches were especially frustrating to us for several reasons. First, they required 15 to 30 minutes each, and during the search eliminated one laboratory assistant who would otherwise test request specimens and answer phone calls for other patients. Second, because the physician often did not know the date on which the test was completed, searches for numerous days on each side of a hypothetical date were required. There was always the nagging doubt that one did not search back far enough when no results were found. Third, it not infrequently turned out that for whatever reason, no specimen had ever been obtained. Finally, we were very confident that the reports of these results were printed and distributed both to the outpatient clinic and to medical records. Something was obviously happening to these reports after they left our area and before they were put on the patient chart. Before the laboratory computer had arrived, paper flowed poorly in the ambulatory care area. After arrival of the computer the paper flow was still poor. The fact that it was now printed by computer is irrelevant. Investigation of the flow of paper distal to the laboratory and into the ambulatory care area disclosed a process involving 11 steps and five persons from four separate areas (Figure 1). The net effect was that the structure of ambulatory care areas had an adverse effect on how well the clinical laboratory could function to serve patient care. It was as if there was a disease in the way that paper flowed in the ambulatory care area. The symptom of it was excessive phone calls for test results from months ago. The clinical laboratory had no other choice than to treat the symptom and ignore the disease. Our treatment consisted of designing and implementing an on-line archive for laboratory results.

We have incorporated an option in the program so that each time a result inquiry transaction is initiated on the computer, the location of the outpatient is automatically captured and placed in a separate file. By examination of this file we can identify those clinics having the most severe problems with paper flow as manifested by frequent calls to the Laboratory Data Center for information that was already sent. Using this management information, we are able to assist the ambulatory care staff in focusing their attention to those clinics that would receive the most benefit from a thorough systems analysis (Figure 2). There are interesting observations that can be made by examining 


\section{Inpatient}

Ambulatory Care

(Result Entered Into Laboratory Computer by Technologist)

$$
\text { (Reports Printed Daily) }
$$

1. Midnight ward clerk picks up reports.

2. Reports charted.
1. Delivered to outpatient tube room.

2. Separate into various front desk locations.

3. P1ace in tube system.

4. Received by front desk on each floor.

5. Separated into various clinic locations.

6. Place in individual clinic boxes.

7. Received by each clinic.

8. Separated into doctor order.

9. Place in individual doctor boxes.

10. Doctor reviews and signs report.

11. Report placed on chart.

\section{SUMMARY}

$\begin{array}{llr}\text { Number of Areas } & 1 & 4 \\ \text { Number of People } & 1 & 5 \\ \text { Number of Steps } & 2 & 11\end{array}$

Figure 1. Comparison of paper-flow distal to laboratory computer for hospitalized and ambulatory care patients.

this study in greater detail. First, by storing both the number of result inquiry and test request transactions by location, one is able to calculate a ratio (RI/TR) that roughly measures the number of telephone calls per test result. This can be used as an index of how well information is handled by each clinic. In the best possible case there would be no calls from the clinic since the desired laboratory test results are already on the chart and reviewed by the clinician. It would be reasonable to assume that if the test results were never available (i.e., the worst possible case), the ratio would be approximately 1.0.- that is, one call for each test result. It was a surprise to discover that there were 


Medical Chief of Clinic
Student Health Service
Renal
Hematology/Oncology
Urology
Surgery
Pediatric Walk In
Medical Walk In
Gynecology
Pediatric
Medicine
Emergency Room
Dermatology
Urology - Private
Opthalmology

$\begin{array}{rrc}38 & 10 & 3.8 \\ 15 & 5 & 3.0 \\ 48 & 69 & .696 \\ 42 & 67 & .627 \\ 25 & 43 & .581 \\ 39 & 72 & .542 \\ 41 & 83 & .494 \\ 30 & 63 & .476 \\ 52 & 137 & .380 \\ 33 & 116 & .284 \\ 41 & 158 & .259 \\ 77 & 457 & .171 \\ 5 & 75 & .067 \\ 0 & 32 & 0.0 \\ 0 & 59 & 0.0\end{array}$

Figure 2. Laboratory usage by clinic. These data were collected by a computer program that analyzes the number of computer transactions for each day. The numbers given are the average number per week measured from June 27 to July 25,1981 . The RI/TR ratio represents the number of phone inquiries per test request and is a useful index of how well each clinic is able to handle the information it receives.

several locations where three to four telephone calls were made for each test request. It is difficult to understand how things could be four times worse than the worst possible case until a thorough review of the entire order entry-result inquiry process is done. For example, in the case of the Student Health Service it was discovered that there is a whole series of problems, including difficulty in establishing proper patient identification, specimen and report delivery times which are not coordinated with the laboratory work cycle, and lack of audit trails in the clinic to establish the proper clinician responsible for the case. The second point of interest is that there are several outpatient locations where the acute nature of patients illness would suggest that frequent telephone inquiry is appropriate. For example, renal clinic, hematology/oncology clinic, and emergency room are in this category. The RI/TR ratio in the first two instances was .696 and .627 , which is close to the usual RL/TR ratio of .75 for intensive care units. The emergency room, however, with the highest number of test results of all, has a ratio of only .171 . The explanation for this low ratio is that there is a remote printer located in the emergency room. As soon as the test result has been completed in the laboratory, it is immediately available, and telephone calls are usually unnecessary. This observation is very important because it gives an indication of the expected drop in calls when a terminal is located in a high-volume stat location such as an intensive care unit or an emergency room. Using the data from our study we estimate that there will be a $75-80 \%$ drop in calls to the laboratory for results when terminals are located on patient units. We have been unable to find other published studies that have quantitated the effect of remote inquiry. This 
also gives an indication of the method by which inefficient paper flow can be eliminated (i.e., terminals on nursing stations and clinics). Finally, it is interesting to compare the RI/TR ratio for the urology clinic (.581) and the private urology clinic (.0). The main difference between these clinics is that the private patients are seen by the faculty clinicians, while house officers are primarily responsible for patients in the former clinic. The large difference in telephone-calling patterns may be a reflection of the fact that physicians in training utilize the laboratory more than experienced clinicians and would be more anxious to know the previous test results when seeing a patient.

The main purpose of laboratory computerization is to enhance communication of test results from the laboratory to the physician. For the hospitalized patient this enhancement is in the form of faster communication by shortening the laboratory turnaround time and speeding up the physician order cycle. ${ }^{\prime}$ For ambulatory care patients, on the other hand, speed is not such a critical issue except in certain areas such as the emergency room. Of much greater importance is the assurance that the report will be present on the chart when the patient returns to clinic. Unlike the hospital setting, where the clinical laboratory can assure that the report is on the ward or in the chart by having its own employees perform this function, delivery of reports each day to 50 outpatient locations spread over a 1-mile area is currently beyond the capacity of the clinical laboratory. Instead, these reports are handled by outpatient clerical personnel. Computerization of the laboratory will not enhance communication of test results to the ambulatory care area unless communication is improved either by a more effective means of handling paper or by conversion to electronic communication via an ambulatory care computer system. The on-line archive system in the laboratory computer is an interim step that treats the symptom but not the disease. More powerful medicine is needed to cure the disease. The medical center is currently testing a computer system for ambulatory care.

Several articles have been published about archive systems for clinical laboratory data. One discussed creation of a file on a hospital computer that is periodically updated by magnetic tapes from the laboratory computer. ${ }^{3}$ Most discuss conversion of the paper archive of a laboratory computer to microfische, either chronologically or by monthly resorting of a large file. ${ }^{4-7}$ Some incorporate an on-line index into the microfische. ${ }^{4,7} \mathrm{We}$ previously tried to solve our ambulatory care phone call problems by converting the daily paper archive of our system to microfische by sending magnetic tapes to an outside bureau every day. This eliminated tome-lifting and much page-turning, but the basic problem was that the callers did not know the collection date of the specimen, much less the date of completion of the test. To remedy this we wrote a program to produce a monthly summary of all laboratory activity for each patient by date. However, this still required a large amount of time to retrieve results from months ago. Until we implemented the on-line archive system for laboratory results on our own laboratory computer, the problem of phone calls from ambulatory care areas for old results was not resolved. Creation of an on-line indexing system would not have required significantly less programming.

Telephone-calling patterns are only one aspect of the difference in needs of ambulatory care areas from the hospital. Other items include the timing, frequency, and format of printed reports. For example, we initially produced interim reports several times per day for both ambulatory care and hospital, until we were informed by the former that they did not wish to receive results quite so fast, and the additional paper was confusing. 
Also we have received numerous comments from physicians in the ambulatory care area that the cumulative summary reports are not particularly helpful to them since the most current results are buried in a large report and are hard to locate. Further, updated reports tend to accumulate in charts instead of being replaced, and the additional paper is confusing. For hospital areas this problem is not noted.

We are currently in the process of upgrading our system and will replace the 55megabyte drives with 200 -megabyte drives. We will then have the capability of storing results on-line for the entire lifetime of the laboratory computer, although a 6- to 12month archive seems to be the optimum amount of storage time required to serve ambulatory care areas. The thought of being able to have all laboratory results immediately available is attractive for research purposes, and we predict that this amount of storage capability will be a common feature of laboratory computer systems in the next few years, especially since the technology is already available and prices continue to drop for hardware.

In summary, when designing a laboratory computer system it is important to recognize that the ambulatory care area has needs and problems that are quite different from inpatient areas. This fact should be recognized and incorporated into the design. One should not expect the same programs, reports, and printing frequency to suffice for both areas. Small differences may have huge impact on the success of the computer in the laboratory, as well as in the hospital and in ambulatory care areas.

\section{REFERENCES}

1. Bloch, D.M., Computer-generated management tools for the clinical pathology laboratory. I Throughput Report. J. Med. Syst. 4:367-380, 1980.

2. Groves, W.E., A dynamic computer system for the clinical laboratory. Am. J. Med. Technol. 44:575$581,1978$.

3. Soffer, S., Entine, S., Wolfe, T., Eggert, A., and Friedman, R., Archival storage of clinical laboratory data. Clin. Chem. 24:436-441, 1978.

4. Litzkow, L., Ingram, W., and Lezott, D., The evolution of a functional real-time laboratory records retrieval and archival system. J. Med. Syst. 1:177-180, 1977.

5. McVittie, J.D., Whitehouse, C., and Wilkinson, R. H., Microfische as a medium for the long-term storage of laboratory computer records. J. Clin. Pathol. 34:49-53, 1981.

6. Pryor, L. R., and Freeman, V. D., An archival system for clinical laboratory data. Am. J. Clin. Pathol. 72:1013-1017, 1979.

7. Roth, E., Solving the record storage problem. Med. Lab. Observer December: 49-53, 1979. 